\title{
ON A NEW SOLUTION OF FRACTIONAL DIFFERENTIAL EQUATION USING COMPLEX TRANSFORM IN THE UNIT DISK
}

\author{
Rabha W. Ibrahim ${ }^{1}$ and Maslina Darus ${ }^{2}$ \\ ${ }^{1}$ Institute of Mathematical Sciences \\ University Malaya, 50603, Kuala Lumpur, Malaysia \\ ${ }^{2}$ School of Mathematical Sciences, Faculty of science and Technology \\ University Kebangsaan Malaysia, Bangi 43600, Selangor Darul Ehsan, Malaysia \\ rabhaibrahim@yahoo.com, maslina@ukm.my
}

\begin{abstract}
The Möbius transform of fractional differential equation (Riccati type) is employed to construct new exact solutions for some nonlinear fractional differential equations. The fractional operators are taken in sense of the modified Srivastava-Owa fractal in the unit disk. Examples are illustrated for problems in biology, economic and physics.
\end{abstract}

Keywords- Fractional calculus, fractional differential equations, Srivastava-Owa fractional operators, unit disk

\section{INTRODUCTION}

Transform method is a mathematical technique that is applied in various fields, such as quantum mechanics, nuclear physics and atomic physics. This technique generates the solutions of partial differential equations; relates solutions of difficult partial differential equations to well known equations and applies to integrable equations. For example, Riccati equation is employed to construct generalized solutions for ordinary and partial differential equations. Various practical transforms for solving various problems were materialized in open literature, such as the Laplace transform, the Fourier transform, the traveling wave transform, the Bäcklund transformation, the integral transform, the fractional integral transforms, the fractional complex transform and Mellin transform.

Fractional differential equations are viewed as option models to nonlinear differential equations. Varieties of them play important roles and tools, not only in mathematics but also in physics, dynamical systems, control systems and engineering, to create the mathematical modeling of many physical phenomena. Furthermore, they are employed in social science such as food supplement, climate and economics. Fractional differential equations concerning the Riemann-Liouville fractional operators or Caputo derivative have been recommended by many authors (see [1-6]). Determining approximate, numerical and exact solutions for fractional differential equations plays a significant role. Numerical solutions or analytic solutions are typically difficult to be computed. It is therefore, required to impose a process to solve the problem of nonlinear fractional differential equations. Recently, one of the most essential and useful methods for fractional calculus appeared as complex fractional transform (integral and derivative) [7-11]. Fractional transform is devised to renovate the fractional differential equations 
into ordinary differential equations, yielding the solution procedure tremendously simple.

In this paper we shall use the Möbius transform of fractional differential equation (Riccati type) to construct the exact solutions for some nonlinear fractional differential equations. The fractional operators are taken in sense of Srivastava-Owa fractal in the unit disk. Examples are illustrated to explain the solution procedure including problems in complex domain.

In [12], Srivastava and Owa, provided the definitions for fractional operators (derivative and integral) in the complex z-plane $\mathrm{C}$ as follows:

Definition 1.1 The fractional derivative of order $\alpha$ is defined, for a function $f(z)$ by

$$
D_{z}^{\alpha} f(z):=\frac{1}{\Gamma(1-\alpha)} \frac{d}{d z} \int_{0}^{z} \frac{f(\zeta)}{(z-\zeta)^{\alpha}} d \zeta ; 0 \leq \alpha<1,
$$

where the function $f(z)$ is analytic in simply-connected region of the complex z-plane $\mathrm{C}$ containing the origin and the multiplicity of $(z-\zeta)^{-\alpha}$ is removed by requiring $\log (z-\zeta)$ to be real when $(z-\zeta)>0$.

Definition 1.2 The fractional integral of order $\alpha$ is defined, for a function $f(z)$, by

$$
I_{z}^{\alpha} f(z):=\frac{1}{\Gamma(\alpha)} \int_{0}^{z} f(\zeta)(z-\zeta)^{\alpha-1} d \zeta ; \alpha>0,
$$

where the function $f(z)$ is analytic in simply-connected region of the complex z-plane (C) containing the origin and the multiplicity of $(z-\zeta)^{\alpha-1}$ is removed by requiring $\log (z-\zeta)$ to be real when $(z-\zeta)>0$.

Remark 1.1 From Definitions 1.1 and 1.2, we have

$$
D_{z}^{\alpha} z^{\beta}=\frac{\Gamma(\beta+1)}{\Gamma(\beta-\alpha+1)} z^{\beta-\alpha}, \beta>-1 ; 0 \leq \alpha<1
$$

and

$$
I_{z}^{\alpha} z^{\beta}=\frac{\Gamma(\beta+1)}{\Gamma(\beta+\alpha+1)} z^{\beta+\alpha}, \beta>-1 ; \alpha>0 .
$$

In our tex, we shall use the following operator:

Definition 1.3 The modified fractional derivative of order $\alpha$ is defined, for a function $f(z)$ by

$$
D_{z}^{\alpha} f(z):=\frac{1}{\Gamma(1-\alpha)} \frac{d}{d z} \int_{0}^{z} \frac{f(\zeta)-f(0)}{(z-\zeta)^{\alpha}} d \zeta ; 0 \leq \alpha<1,
$$

where the function $f(z)$ is analytic in simply-connected region of the complex z-plane $\mathrm{C}$ containing the origin and the multiplicity of $(z-\zeta)^{-\alpha}$ is removed by requiring $\log (z-\zeta)$ to be real when $(z-\zeta)>0$.

Note that Definition 1.3 satisfies the analytic function of the form

$$
f(z)=\sum_{n=1}^{\infty} a_{n} z^{n}, \quad z \in \mathrm{C}
$$


This class of analytic functions has wide applications in the geometric function theory and univalent function theory when $a_{1}=1$.

Recently, the authors employed these operators widely in the geometric function theory by extending some classes of analytic functions into classes of analytic functions of fractional power (see [13-16]).

Note that the real case of the Srivastava and Owa fractional operators coincides with the Riemann-Liouville fractional operator which are given by the following definition.

Definition 1.4 The fractional (arbitrary) order integral of the function $f$ of order $\alpha>0$ is defined by

$$
I_{a}^{\alpha} f(t)=\int_{a}^{t} \frac{(t-\tau)^{\alpha-1}}{\Gamma(\alpha)} f(\tau) d \tau,
$$

When $a=0$, we write $I_{0}^{\alpha} f(t)=I^{\alpha} f(t)$. It was shown that $I_{a}^{\alpha} f(t)=f(t) * \phi_{\alpha}(t)$, where $(*)$ denoted the convolution product (see [1]), $\phi_{\alpha}(t)=\frac{t^{\alpha-1}}{\Gamma(\alpha)}, t>0$ and $\phi_{\alpha}(t)=0, t \leq 0$ and $\phi_{\alpha} \rightarrow \delta(t)$ as $\alpha \rightarrow 0$ where $\delta(t)$ is the delta function.

Definition 1.5 The fractional (arbitrary) order derivative of the function $f$ of order $0 \leq \alpha<1$ is defined by

$$
D_{a}^{\alpha} f(t)=\frac{d}{d t} \int_{a}^{t} \frac{(t-\tau)^{-\alpha}}{\Gamma(1-\alpha)} f(\tau) d \tau=\frac{d}{d t} I_{a}^{1-\alpha} f(t) .
$$

Remark 1.2 From Definition 1.4 and Definition 1.5, $a=0$, we have

and

$$
D^{\alpha} t^{\mu}=\frac{\Gamma(\mu+1)}{\Gamma(\mu-\alpha+1)} t^{\mu-\alpha}, \mu>-1 ; 0<\alpha<1
$$

$$
I^{\alpha} t^{\mu}=\frac{\Gamma(\mu+1)}{\Gamma(\mu+\alpha+1)} t^{\mu+\alpha}, \mu>-1 ; \alpha>0
$$

\section{TRANSFORM METHOD}

The fractional Riccati equation in complex domain takes the form:

$$
D_{\xi}^{\alpha} \mu(\xi)=\sigma+\mu^{2}(\xi), \quad \alpha \in(0,1]
$$

where $\sigma$ is a real constant and $\mu: U \rightarrow \mathrm{C} ; U:=\{z \in \mathrm{C},|z| \leq 1\}$. It is well known that the solution of (1) takes the form in terms of the generalized hyperbolic and trigonometric functions (see [1]) 


$$
\mu(\xi)= \begin{cases}-\sqrt{-\sigma} \tanh _{\alpha}(\sqrt{-\sigma} \xi) & \text { for } \sigma<0 \\ -\sqrt{-\sigma} \operatorname{coth}_{\alpha}(\sqrt{-\sigma} \xi) & \text { for } \sigma<0 \\ \sqrt{\sigma} \tan _{\alpha}(\sqrt{\sigma} \xi) & \text { for } \sigma>0 \\ -\sqrt{\sigma} \cot _{\alpha}(\sqrt{\sigma} \xi) & \text { for } \sigma>0 \\ -\frac{\Gamma(1+\alpha)}{\xi^{\alpha}+v} & \text { for } \sigma=0,\end{cases}
$$

where $v$ is a constant. We shall assume the complex fractional differential equation with independent variable $\left(t, z_{1}, \ldots, z_{j}\right)$ and dependent variable $u$

$$
F\left(u, u_{t}, u_{z_{1}}, \ldots, u_{z_{j}}, u_{z_{1} z_{1}}, \ldots, D_{z_{1}}^{\alpha} u, \ldots, D_{z_{j}}^{\alpha} u, \ldots\right)=0,
$$

where $D_{z_{1}}^{\alpha}, \ldots, D_{z_{j}}^{\alpha}$ are the modified Srivastava-Owa fractals.

Our method can be summarized as follows:

Step 1: Using the complex wave transform

$$
\begin{gathered}
u\left(t, z_{1}, \ldots, z_{j}\right)=u(\eta), \\
\eta=\eta_{0}+\lambda t+\lambda_{1} z_{1}+\ldots+\lambda_{j} z_{j},
\end{gathered}
$$

where $\lambda, \lambda_{i}(i=1, \ldots, j)$ are constants. Eq.(3) becomes

$$
\Phi\left(u(\eta), u^{\prime}(\eta), u^{\prime \prime}(\eta), \ldots, D_{\eta}^{\alpha} u\right)=0,
$$

where $\left({ }^{\prime}\right)=\frac{d}{d \eta}$.

Step 2: Assuming that (4) has a solution of the form

$$
u(\eta)=\sum_{m=0}^{n} a_{m} \phi^{m}(\eta)
$$

where $a_{m}(m=0, \ldots, n)$ are constants to be calculated and $\phi$ computes from the Möbius transform

$$
\phi(\eta)=\frac{A \mu(\eta)+\sigma}{B \mu(\eta)+\sigma}
$$

where $A \neq B$ and $\sigma \neq 0$.

Step 3: Substituting (5) in (4) and setting the coefficients of the powers of $\phi$ to be zero, we impose a nonlinear algebraic system in $a_{m}$ and $\lambda$.

Step 4: Solving the system to obtain these values and substituting them into Eq.(5) we may receive the exact solutions of (3).

\section{APPLICATIONS}

In this section we shall illustrate two examples to examine our method.

\subsection{Example}

Water as a liquid moves through the vadose region in response to gravity and 
gradients of pressure. Recall that the vadose region has hole spaces filled with both air and liquid water. The water pressure depends on the water saturation and related capillary forces. Because the soil is only partially saturated the pressure is negative due to capillarity. If the soil is uniform in its properties such as composition, capillary pressures are most negative where the soil is dry, and most positive where it is wet. As an FDE it can be represented as

$$
D_{t}^{\alpha} u-\kappa u D_{z}^{\alpha} u-\delta D_{z}^{2 \alpha} u=0,
$$

where $z$ is the position in this model and $u$ is the so-called volumetric water content. It denotes the proportion of the space filled by water. $\delta$ is the so-called soil moisture diffusivity and $\kappa$ is the saturation dependent hydraulic conductivity. Equation (7) describes the infiltration in the vadose region. The advection is due the gravity and the diffusion is due to capillary wicking.

Using the complex wave transform

$$
u(t, z)=u(\eta), \quad \eta=\lambda t+z
$$

we receive

$$
\lambda^{\alpha} D_{\eta}^{\alpha} u-\kappa u D_{\eta}^{\alpha} u-\delta D_{\eta}^{2 \alpha} u=0 .
$$

By applying the above method yields

$$
u(\eta)=a_{0}+a_{1} \frac{A \mu(\eta)+\sigma}{B \mu(\eta)+\sigma}
$$

such that

$$
a_{0}=\frac{\lambda^{\alpha}}{\kappa}, \quad a_{1}=\frac{-2 \delta}{\kappa}, \kappa \neq 0
$$

and $\mu$ is the solution of Riccati equation defined in (2). Now for $\sigma<0$, we impose the solutions

$$
u(\eta)=\frac{\lambda^{\alpha}}{\kappa}+\left(\frac{-2 \delta}{\kappa}\right) \frac{A\left[-\sqrt{-\sigma} \tanh _{\alpha}(\sqrt{-\sigma} \eta)\right]+\sigma}{B\left[-\sqrt{-\sigma} \tanh _{\alpha}(\sqrt{-\sigma} \eta)\right]+\sigma}
$$

and

$$
u(\eta)=\frac{\lambda^{\alpha}}{\kappa}+\left(\frac{-2 \delta}{\kappa}\right) \frac{A\left[-\sqrt{-\sigma} \operatorname{coth}_{\alpha}(\sqrt{-\sigma} \eta)\right]+\sigma}{B\left[-\sqrt{-\sigma} \operatorname{coth}_{\alpha}(\sqrt{-\sigma} \eta)\right]+\sigma}
$$

While for $\sigma>0$ implies the solutions

and

$$
u(\eta)=\frac{\lambda^{\alpha}}{\kappa}+\left(\frac{-2 \delta}{\kappa}\right) \frac{A\left[\sqrt{\sigma} \tan _{\alpha}(\sqrt{\sigma} \eta)\right]+\sigma}{B\left[\sqrt{\sigma} \tan _{\alpha}(\sqrt{\sigma} \eta)\right]+\sigma}
$$

$$
u(\eta)=\frac{\lambda^{\alpha}}{\kappa}+\left(\frac{-2 \delta}{\kappa}\right) \frac{A\left[-\sqrt{\sigma} \cot _{\alpha}(\sqrt{\sigma} \eta)\right]+\sigma}{B\left[-\sqrt{\sigma} \cot _{\alpha}(\sqrt{\sigma} \eta)\right]+\sigma} .
$$

solutions of Eq.(8). 


\subsection{Example}

In 1973, Fischer Black and Myron Scholes [17] suggested the famous theoretical valuation formula for options. The main fictional idea of Black and Scholes excites in the texture of a riskless portfolio taking positions in bonds (cash), option and the underlying stock. Such an process strengthens the use of the no-arbitrage principle as well. The Black-Scholes model for the value of an option can be described by the fractional equation

$$
D_{t}^{\alpha} u+\rho(t, z) D_{z}^{2 \alpha} u+u D_{z}^{\alpha} u-r(t, z) u=0, \quad t \in(0, T)
$$

where $u(t, z)$ is the European call option price at asset price $z$ (positive real number) and at time $t ; r(t, z)$ is the risk free interest rate, and $\rho(t, z)$ represents the volatility function of underlying asset. By employing the wave transform

$$
u(t, z)=u(\eta), \quad \eta=\lambda t+\lambda_{1} z
$$

we extradite

$$
\lambda^{\alpha} D_{\eta}^{\alpha} u+\lambda_{1}^{2 \alpha} \rho(\eta) D_{\eta}^{2 \alpha} u+\lambda_{1}^{\alpha} u D_{\eta}^{\alpha} u-r(\eta) u=0 .
$$

Now in virtue of the above method, we have

$$
a_{0}=\frac{-\lambda^{\alpha} \sigma}{\lambda_{1}^{\alpha} \sigma-r}, \quad a_{1}=\frac{-2 \lambda_{1}^{2 \alpha} \rho \sigma}{\lambda_{1}^{\alpha} \sigma-r},
$$

where $\lambda_{1}^{\alpha} \sigma \neq r$. For $\sigma<0$, we impose the solutions

$$
u(\eta)=\frac{-\lambda^{\alpha} \sigma}{\lambda_{1}^{\alpha} \sigma-r}+\left(\frac{-2 \lambda_{1}^{2 \alpha} \rho \sigma}{\lambda_{1}^{\alpha} \sigma-r}\right) \frac{A\left[-\sqrt{-\sigma} \tanh _{\alpha}(\sqrt{-\sigma} \eta)\right]+\sigma}{B\left[-\sqrt{-\sigma} \tanh _{\alpha}(\sqrt{-\sigma} \eta)\right]+\sigma}
$$

and

$$
u(\eta)=\frac{-\lambda^{\alpha} \sigma}{\lambda_{1}^{\alpha} \sigma-r}+\left(\frac{-2 \lambda_{1}^{2 \alpha} \rho \sigma}{\lambda_{1}^{\alpha} \sigma-r}\right) \frac{A\left[-\sqrt{-\sigma} \operatorname{coth}_{\alpha}(\sqrt{-\sigma} \eta)\right]+\sigma}{B\left[-\sqrt{-\sigma} \operatorname{coth}_{\alpha}(\sqrt{-\sigma} \eta)\right]+\sigma} .
$$

While for $\sigma>0$ implies the solutions

and

$$
u(\eta)=\frac{-\lambda^{\alpha} \sigma}{\lambda_{1}^{\alpha} \sigma-r}+\left(\frac{-2 \lambda_{1}^{2 \alpha} \rho \sigma}{\lambda_{1}^{\alpha} \sigma-r}\right) \frac{A\left[\sqrt{\sigma} \tan _{\alpha}(\sqrt{\sigma} \eta)\right]+\sigma}{B\left[\sqrt{\sigma} \tan _{\alpha}(\sqrt{\sigma} \eta)\right]+\sigma}
$$

$$
u(\eta)=\frac{-\lambda^{\alpha} \sigma}{\lambda_{1}^{\alpha} \sigma-r}+\left(\frac{-2 \lambda_{1}^{2 \alpha} \rho \sigma}{\lambda_{1}^{\alpha} \sigma-r}\right) \frac{A\left[-\sqrt{\sigma} \cot _{\alpha}(\sqrt{\sigma} \eta)\right]+\sigma}{B\left[-\sqrt{\sigma} \cot _{\alpha}(\sqrt{\sigma} \eta)\right]+\sigma} .
$$

solutions of Eq.(10).

\subsection{Example}

A drainage equation is an equation characterizing the relevance between depth and spacing of parallel subsurface drains, depth of the water table, depth and hydraulic conductivity of the soils. It is employed in drainage design which reads for fractional time-space as follows: 


$$
\begin{gathered}
D_{t}^{\alpha} u=\frac{1}{2} u D_{z}^{2 \alpha} u-2 u^{2} D_{z}^{\alpha} u+\left(D_{z}^{\alpha} u\right)^{2}, \\
(t \in(0, T), z \in U, 0<\alpha \leq 1) .
\end{gathered}
$$

The foam drainage equation is a pattern of the flow of liquid through channels and nodes (intersection of four channels) between the bubbles, driven by gravity and capillarity [18]. Now by using the complex transform

$$
u(t, z)=u(\eta), \quad \eta=z+\lambda t
$$

where $\lambda$ is constant. Substituting (12) into Eq. (11), we receive the fractional ordinary differential equation:

$$
\lambda^{\alpha} D_{\eta}^{\alpha} u=\frac{1}{2} u D_{\eta}^{2 \alpha} u-2 u^{2} D_{\eta}^{\alpha} u+\left(D_{\eta}^{\alpha} u\right)^{2}
$$

By balancing the highest order derivative terms and nonlinear terms in Eq. (13), we assume that Eq. (13) have the following formal solution

$$
u(\eta)=a_{0}+a_{1} * \frac{A \mu(\eta)+\sigma}{B \mu(\eta)+\sigma}
$$

where $\mu(\eta)$ satisfies Eq. (1).

Substituting Eq. (14) along with Eq. (1) into Eq. (13) and then setting the coefficients of $\mu(\eta)$ to zero, we can impose a set of algebraic equations about $a_{0}, a_{1}$ and $\sigma$. Solving the algebraic equations yielding

$$
a_{0}=0, \quad a_{1}=1, \quad \sigma=\psi(\lambda)
$$

where $\psi$ is a linear function in $\lambda^{\alpha}, \lambda>0$. Substituting the above assertion into (14), implies new types of exact solutions of Eq. (11) as follows:

For $\sigma<0$, we impose the solutions

$$
u(\eta)=\frac{A\left[-\sqrt{-\sigma} \tanh _{\alpha}(\sqrt{-\psi(\lambda)} \eta)\right]+\psi(\lambda)}{B\left[-\sqrt{-\psi(\lambda)} \tanh _{\alpha}(\sqrt{-\psi(\lambda)} \eta)\right]+\psi(\lambda)}
$$

and

$$
u(\eta)=\frac{A\left[-\sqrt{-\psi(\lambda)} \operatorname{coth}_{\alpha}(\sqrt{-\psi(\lambda)} \eta)\right]+\psi(\lambda)}{B\left[-\sqrt{-\psi(\lambda)} \operatorname{coth}_{\alpha}(\sqrt{-\psi(\lambda)} \eta)\right]+\psi(\lambda)} .
$$

while for $\sigma>0$, the solutions

$$
u(\eta)=\frac{A\left[\sqrt{\psi(\lambda)} \tan _{\alpha}(\sqrt{\psi(\lambda)} \eta)\right]+\psi(\lambda)}{B\left[\sqrt{\psi(\lambda)} \tan _{\alpha}(\sqrt{\psi(\lambda)} \eta)\right]+\psi(\lambda)}
$$

and

$$
u(\eta)=\frac{A\left[-\sqrt{\psi(\lambda)} \cot _{\alpha}(\sqrt{\psi(\lambda)} \eta)\right]+\psi(\lambda)}{B\left[-\sqrt{\psi(\lambda)} \cot _{\alpha}(\sqrt{\psi(\lambda)} \eta)\right]+\psi(\lambda)} .
$$




\section{CONCLUSION}

From above we conclude that the Möbius transform of fractional differential equation (Riccati type) affected on the exact solutions of fractional differential equations in complex domain. The fractional operators are taken in senses of the Srivastava-Owa fractional operators and the Riemann-Liouville fractional operators. We applied the proposed method on different types of fractional differential equations, such as liquid movement equation, Black-Scholes fractional differential equation and fractional drainage equation in order to create new exact solutions.

Acknowledgement- The second author is fully supported by LRGS/TD/2011/UKM/ICT/03/02.

\section{REFERENCES}

1. I. Podlubny, Fractional Differential Equations, Acad. Press, London, 1999.

2. B. J. West, M. Bologna, and P. Grigolini, Physics of Fractal Operators, Institute for Nonlinear Science, Springer, New York, NY, USA, 2003.

3. A. A. Kilbas, H. M. Srivastava, and J. J. Trujillo, Theory and Applications of Fractional Differential Equations, vol. 204 of North-Holland Mathematics Studies, Elsevier Science B.V., Amsterdam, The Netherlands, 2006.

4. J. Sabatier, O. P. Agrawal, and J. A. Machado, Advance in Fractional Calculus: Theoretical Developments and Applications in Physics and Engineering, Springer, Dordrecht, The Netherlands, 2007.

5. V. Lakshmikantham, S. Leela, J. Vasundhara, Theory of Fractional Dynamic Systems. Cambridge Academic Publishers, Cambridge 2009.

6. D. Baleanu, B. Guvenc and J. A. Tenreiro, New Trends in Nanotechnology and Fractional Calculus Applications, Springer, New York, NY, USA, 2010.

7. Z.B. Li, J.H. He, Application of the fractional complex transform to fractional differential equations, Nonlinear Science Letters A 2, 121-126, 2011.

8. R. W. Ibrahim, Fractional complex transforms for fractional differential equations, Advances in Difference Equations 2012, 2012:192 doi:10.1186/1687-1847-2012-192.

9. R. W. Ibrahim, Complex transforms for systems of fractional differential equations, Abstract and Applied Analysis 2012, Article ID 814759, 15 pages.

10. H. M. Srivastava, M. Darus, and R. W. Ibrahim, Classes of analytic functions with fractional powers defined by means of a certain linear operator, Integral Transforms and Special Functions 22,17-28, 2011.

11. S. Sivasubramanian, M. Darus, R. W. Ibrahim, On the starlikeness of certain class of analytic functions, Mathematical and Computer Modelling 54(1-2), 112-118, 2011.

12. H.M. Srivastava, S. Owa, Univalent Functions, Fractional Calculus, and Their Applications, Halsted Press, John Wiley and Sons, New York, Chichester, Brisban, and Toronto, 1989.

13. R.W. Ibrahim and M. Darus, Differential operator generalized by fractional derivative, Miskolc Mathematical Notes 12, 167-184, 2011.

14. R.W. Ibrahim and M. Darus, Subordination and superordination for univalent solutions for fractional differential equations, Journal of Mathematical Analysis and 
Applications 345, 871-879, 2008.

15. R. W. Ibrahim, M. Darus, Subordination and superordination for analytic functions involving fractional integral operator, Complex Variables and Elliptic Equations 53, 1021-1031, 2008.

16. R. W. Ibrahim, M. Darus, On Analytic functions associated with the Dziok-Srivastava linear operator and Srivastava-Owa fractional integral operator, Arabian Journal for Science and Engineering 36, 441-450, 2011.

17. F. Black, M. S. Scholes, The pricing of options and corporate liabilities, Journal of Political Economy 81, 637-654, 1973.

18. D. Weaire, S. Hutzler, S. Cox, M.D. Alonso, D. Drenckhan, The Fluid Dynamics of Foams, Journal of Physics: Condensed Matter 15, 65-73, 2003. 